\title{
Reply \\ Effect of Divalproex Combined with Olanzapine or Risperidone in Patients with an Acute Exacerbation of Schizophrenia
}

\author{
Daniel E Casey*, 1,2, David G Daniel ${ }^{2,3}$, Adel A Wassef ${ }^{4}$, Latherine A Tracy ${ }^{5,6}$, Patricia Wozniak ${ }^{5}$ and Kenneth \\ W Sommerville ${ }^{5}$, For the Depakote psychosis group \\ 'Portland VA Medical Center, Portland, OR, USA; ${ }^{2}$ George Washington Univesity, DC, USA; ${ }^{3}$ Bioniche Development, Folls Church, VA, USA; \\ ${ }^{4}$ HCPC-University of texas, Houston, TX, USA; ${ }^{5}$ Abbott Laboratories, Abbott Park, IL, USA; ${ }^{6}$ University of Illinois, Chicago, IL, USA
}

Neuropsychopharmacology (2003) 28, 2052-2053, advance online publication, 16 July 2003; doi: I 0. I 038/sj.npp. 1300246

Sir

In his comment on Casey et al (2003), Winterer has offered two major criticisms of the paper. First, he argues that evidence suggests that valproate at therapeutic doses likely does not produce physiological effects in brain GABA that account for the mechanism of the therapeutic benefits of adding valproate to atypical antipsychotics. Second, Winterer argues that there is evidence for multiple other mechanisms that may underlie the therapeutic benefits of valproate add-on to atypicals in schizophrenia.

Of course, any suggestion with regard to the mechanism of action for the therapeutic benefit of valproate add-on in schizophrenia qualifies only as speculation. However, in support of his first argument, Winterer sites a review (Winterer and Herrmann, 2000) which focuses on the benefits of valproate for agitated and aggressive behaviors in schizophrenia and on basic science studies investigating temporal lobe GABA (mostly centered on epilepsy) activity following the administration of therapeutic and supratherapeutic doses of valproate. Valproate-induced GABA inhibitory potentials in these temporal structures were mostly observed at supratherapeutic doses. However, in the Casey et al clinical study, the results showed that divalproex add-on was beneficial for core psychotic symptoms such as delusions, unusual thought content, and difficulty in abstract thinking. These brain functions are controlled

\footnotetext{
*Correspondence: Dr DE Casey, Portland VA Medical Center, Mental Illness Research, Education \& Clinical Centre (P3MIRECC), 3710 SW US Veterens Hospital Road, Portland, OR, USA, Tel: + I 503220 8262 ext 56477, fax: + I 503273521 I,

E-mail: daniel.casey@med.va.gov

${ }^{2} \mathrm{Dr}$ Casey is engaged in research activities supported by Abbott Laboratories.

Received 0I April 2003; accepted 08 May 2003

Online publication: 22 May 2003 at http://www.acnp.org/citations/ Npp52203/39/default.pdf
}

primarily by higher cortical structures such as the cingulate and frontal cortices. Deutch (1992) reports that the prefrontal cortex exerts regulatory control over subcortical structures linked to psychosis. In fact, it is in these higher brain areas that recent cell counting and binding studies have found reductions in GABA interneurons and GABA activity in schizophrenics (Benes, 2000). These inhibitory GABA interneurons are thought to interact with dopamine neurons in modulating output from these higher cortical structures. A number of studies examining the effect of valproate on animal models of psychosis have found significance at therapeutic doses and one study suggested that the effect was due to increased GABA inhibition of dopamine activity (eg Yang et al, 2000). Meltzer et al (2001) found synergistic effects on dopamine for therapeutic doses of valproate and atypical antipsychotics in the prefrontal cortex but not in the nucleus accumbens of rats, likely due to inhibitory GABA modulation. Additionally, Wassef et al (1999) reviewed other GABAergic compounds that have been shown to be effective as add-on for schizophrenia. It seems unlikely that these drugs share a further mechanism that improves psychotic symptoms when added to an antipsychotic.

We would concede Winterer's second point that a number of studies have identified other potential CNS mechanisms of action for valproate, including effects on postreceptor signaling systems, RNA expression, and neuroprotection. As suggested in our prior discussion of these results, it certainly is a possibility that the ability of valproate to add to the efficacy of atypical antipsychotic medicines in schizophrenia is mediated by one of these other mechanisms.

Isaac and Isaac report on an open label trial of valproate combined with atypical antipsychotics $v s$ atypical or typical antipsychotics alone. While they show some promising early results, the study is still ongoing and the small sample sizes to date do not allow definitive conclusions at this time. They do report trends toward shorter lengths of hospitalization with the addition of valproate to atypicals compared 
with atypical or typical antipsychotics alone. Additionally, higher scores on Reward Dependence are reported for the valproate combined with the atypical group $v s$ the atypical group alone. The lack of findings regarding BPRS may be due to the small sample sizes to date and also are confounded by differences in baseline scores across groups that may require covariance analysis. Further interpretation of the preliminary results is also hindered by lack of information on randomization of patients to treatment groups, blood levels for valproate, speed of titration for valproate, and equivalence of dosing for atypicals across groups.

We are intrigued by the early results reported in this study and the success in reducing the length of hospital stays with the addition of valproate. We look forward to a published report on completed data and a full methodological description of this study.

\section{REFERENCES}

Benes FM (2000). Emerging principles of altered neural circuitry in schizophrenia. Brain Res Rev 31: 251-269.
Casey DE, Daniel DG, Wassef AA, Tracy K, Wozniak P, Sommerville KW (2003). Effect of divalproex combined with olanzepine or risperidone in patients with an acute exacerbation of schizophrenia. Neuropsychopharmacology 25: $1-27$.

Deutch AY (1992). The regulation of subcortical dopamine systems by the prefrontal cortex: interactions of central dopamine systems and the pathogenesis of schizophrenia. J Neural Transm 36(Suppl): 61-89.

Meltzer HY, Dai J, Ichikawa J (2001). Valproic Acid, an Anticonvulsant Mood Stabilizer, Potentiates AntipsychoticInduced Dopamine Release in Rat Medial Prefrontal Cortex but not Nucleus Accumbens. Society for Neuroscience: San Diego.

Wassef AA, Dott SG, Harris A, Brown A, O’Boyle M, Meyer (1999). Critical review of GABAergic drugs on the treatment of schizophrenia. J Clin Psychopharmacol 19: 222-232.

Winterer G, Herrmann WM (2000). Valproate and the symptomatic treatment of schizophrenia spectrum patients. Pharmacopsychiatry 33: 182-188.

Yang P, Beasley A, Swann A, Dafny N (2000). Valproate modulates the expression of methylphenidate (Ritalin) sensitization. Brain Res 874: 216-220. 